\title{
Adaptation Oriented "Resource" Modeling for Course-based E-learning Environment
}

\author{
http://dx.doi.org/10.3991/ijet.v9i1.3090 \\ Kalla. Madhu Sudhana ${ }^{1}$, V. Cyril Raj ${ }^{2}$ and T.Ravi ${ }^{3}$ \\ ${ }^{1}$ St. Peter's University, Chennai, India \\ ${ }^{2}$ Dr M.G.R University, Chennai, India \\ ${ }^{3}$ Srinivasa Institute of Engineering \& Technology, Chennai, India
}

\begin{abstract}
During the recent years, the semantic technology such as ontology based representation of metadata has influenced the research, in the area of educational software for accessing, searching and structuring of e-learning contents to provide personalized, adaptive and context aware delivery of learning content. In this paper, we present ontology-based resource description metadata model which is particularly useful for context-aware adaptive e-learning situation. First, we mentioned some of the features to be considered in developing resource description ontology then proposed an ontology called Context-aware Adaptive Learning Resource Ontology (CALRO) that is suitable for delivery of content according to learner contextual information such as learner's learning style and characteristics of the learning device. Finally, we discuss the concerned adaptation approach and its workflow behavior.
\end{abstract}

Index Terms-Ontology-based, Context-aware, Adaptivelearning, Resource description ontology, CALRO

\section{INTRODUCTION}

For modeling and organizing learning resources, the current e-learning standards and platforms have not considered the importance of metadata, up to required satisfaction level to the pedagogical needs, preferences and diversity in learning device characteristics, so as to perform context-aware adaptive and personalized delivery of learning contents.

In e-learning environments the content was adaptively structured to access through various learning devices, as per the device capabilities, communication channel variations and user preferences [1]. Due to the technical diversity in the characteristics of learning devices, there is a need to organize and manage the existing learning resources to access them as per the device capabilities and pedagogical needs of e-learner.

In e-learning domain the key components are: Learner requirements, Learning device and Learning resources. The existing literature has covered various diversity problems in these components such as:

(1) Different learners may prefer to read different types of learning material (Applications, case studies, exercises, etc.).

(2) Due to technical and communication advancements there are various types of learning devices (PC, Cell Phone, PDA, etc.).
(3) Learning resources are available in different forms and characteristics (reports, books, web sites, videos, etc.).

These divergences require developing a metadata that helps to organize learning resources so as to deliver suitable learning content as per the context of e-learner and to present supporting information (resources) which improves learner's understandability and cognitive ability. The metadata must contain extensive features such as examples, applications, references, etc. along with the contextual information.

The proposed ontology with three different features is called as "Context aware Adaptive Learning Resource Ontology (CALRO)", in which various contextual and extensive features have been considered and it is mainly designed for semantically enriched adaptive e-learning system.

This paper presents the need of adaptation oriented resource description model and its required features and then introduced the proposed Context aware Adaptive Learning Resource Ontology that makes the learning resources to index and present, based on their conceptual inter relations, which supports concept based navigation of learning contents. Finally, we discussed the proposed adaptation approach along with its usage and workflow scenario.

\section{RELATED WORK}

The semantic technology and ontological approach are emerging as promising approaches to maximize the retrieval and presentation of dynamic context [2]. The ontological approach is a research domain to overcome the most common problems for intelligent applications in an educational domain [3]. Here, the ontological approach to represent metadata of learning resources supports adaptation oriented resource (Content) modeling that allows us to organize and index resources, so as to deliver suitable content based on concerned context of e-learner.

The resource description meta-data allows searching and retrieval of suitable learning content [4]. Ontology is a knowledge representation approach that describes the knowledge through defining concepts along with their properties and relationships [5] and in [6] it is mentioned that, ontology-based navigation allows the user to obtain an increased degree of constrained navigation based on courseware lesson plans. So that the description of learning resources through ontology makes the adaptation 
PAPER

AdAPTATION ORIENTED “RESOURCE” MODELING FOR COURSE-BASED E-LEARNING ENVIRONMENT

mechanism to access the contents based on their semantic relationships.

In the field of intelligent web based tutoring systems Brusilovsky [7] proposed DCG (Dynamic Course Generation) approach for automatic generation of personalized course contents as per the learner's knowledge and goals.

The open source project called the "EDUTELLA Project" [8] is developed based on association of semantics among the learning resources. In this the Resource Description Framework (RDF) [9] based digital resources is used for the exchange of learning objects and services. AdaptWeb (Adaptive Web-based learning Environment) [10] delivers the learning resources based on traditional student model.

\section{NEED of AdAPTATION ORIENTED RESOURCE MODELING}

The metadata model of the adaptive learning resources must provide an environment to deliver the right resource that satisfies the learner preferences, device capabilities and presentation features (that enriches understandability and cognitive skills of e-learner). The context adaptation module in an adaptive e-learning identifies the current student's context, and then recommends appropriate learning resources accordingly [11]. To enable the identification of required content, the learning resources must be managed and organized with suitable metadata.

The adaption mechanism of e-learning content must address to meet device and user preference needs [12]. An important issue in an adaptive e-learning domain is the discovery of suitable learning resources as per the different contextual and cognitive needs of e-learner so that, the metadata information of e-learning content can be categorized as shown in "Fig. 1".

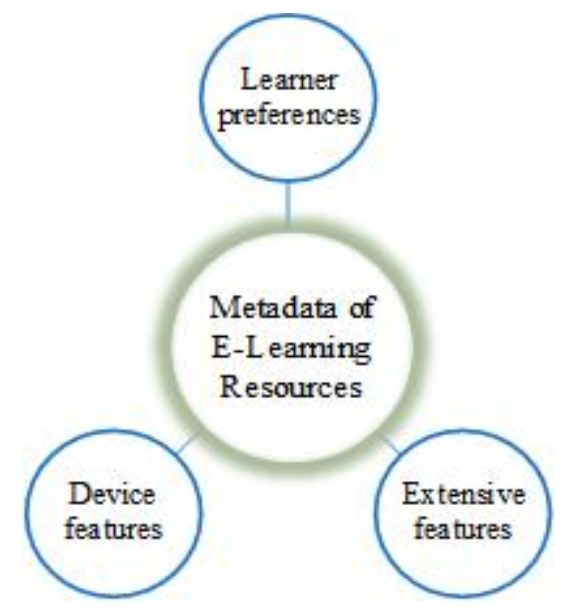

Figure 1. Metadata categories of e-learning content

Device features: Due to technical advancements in communication technology has initiated the change of paradigm from traditional computer based e-learning to m-learning. The diversity of digital learning devices initiated to incorporate device features in learning resource metadata model, which is useful to find suitable content based on device capabilities.

Learner preferences: The learner preference profile may consist of two categories of information such as domain independent and domain specific preferences. The domain independent category includes the personal learning style description of e-learner and domain specific preferences provides the information related to learner's learning topic such as subject, domain, language, etc. The adaptation mechanism uses learner preference profile for personalized delivery of learning contents.

Extensive features: This category of metadata helps to recommend extensive information (resources) to the learner. It improves the understandability, knowledge level and cognitive skills of e-learner. This sort of metadata is useful to deliver contents as per the presentation environment which is designed to study semantically related topics and everything pertaining to particular concept.

\section{Context Aware Adaptive LeARning Resource ONTOLOGY (CALRO)}

As stated by Siadaty [13] the learning context can be referred as, all the information about the situation in which learning is occurred. To build context aware adaptive elearning system, as shown in "Fig. 1" we identified three important features that need to be considered to describe learning content. These three features have been considered in proposed CALRO ontology, which acts as metadata for resource description in context aware adaptive e-learning system.

In order to exchange the semantics of learning resources, the explicitly modeled ontologies provide a way of representing formal and shared information. Thus, the ontological approach can be used to index resources indicating their semantic meaning; thereby resource semantics can be explicit and machine-accessible.

The proposed ontology introduces various metadata features for improving presentation, adaptation and navigation in e-learning environment. CALRO is developed with three different categories of features, where the conceptually related resources may fall within particular classification system.

The resource description ontology (the proposed CALRO) can be formally described with 4-tuples, $<$ Tc, Tf, Ps, Rs $>$. The symbols Tc, Tf, Ps and Rs represent as:

Tc - Topic Class (Base Class)

Tf - Topic Features Set \{Device features, Learner preferences, Extensive features $\}$

Ps - Property Set $\{$ Name, ID, Description, Domain, Alias, Language, Objective $\}$

Rs - Relations Set \{hasTitle, hasID, hasDeviceFeature, hasExtensiveFeature, etc.\}

The ontology is composed of mainly four core classes that encompass different features explained in previous section in order to support semantic-based search and adaptation. Table I, indicates the semantics and purpose of these core classes. The layout of different classes and sub classes relationships of the proposed ontology developed in "Protégé tool [14]" is as shown in "Fig. 2". The details of the topic are specified with properties such as Title (hasTitle), ID (hasID), Course (ofCourse), Description (hasDescription), etc. Some of the data properties and object properties of base class Topic and individuals of sub class Media are shown in "Fig. 3". 
PAPER

AdAPTATION ORIENTED “RESOURCE” MODELING FOR COURSE-BASED E-LEARNING ENVIRONMENT

TABLE I.

DESCRIPTION OF CORE CLASSES IN CALRO ONTOLOGY

\begin{tabular}{|c|c|c|}
\hline $\begin{array}{l}\text { Classes } \\
\text { Name }\end{array}$ & Semantics & Purpose \\
\hline Topic & $\begin{array}{l}\text { Is the root class } \\
\text { of proposed } \\
\text { ontology }\end{array}$ & $\begin{array}{l}\text { The core classes of three } \\
\text { different features indicate the } \\
\text { conceptually related topics of } \\
\text { the base topic. }\end{array}$ \\
\hline Learner-Pref & $\begin{array}{l}\text { It deals with, to } \\
\text { whom the } \\
\text { resource is } \\
\text { useful? }\end{array}$ & $\begin{array}{l}\text { Describes the learning-style } \\
\text { context of e-learner. }\end{array}$ \\
\hline Device-Feat & $\begin{array}{l}\text { To what type of } \\
\text { device it can be } \\
\text { delivered? }\end{array}$ & $\begin{array}{l}\text { This category describes the } \\
\text { context of topic presentation } \\
\text { based on supporting device } \\
\text { characteristics. }\end{array}$ \\
\hline $\begin{array}{l}\text { Extensive- } \\
\text { Feat }\end{array}$ & $\begin{array}{l}\text { What are the } \\
\text { other supporting } \\
\text { resources? }\end{array}$ & $\begin{array}{l}\text { The semantically related topics } \\
\text { of targeted resource, for further } \\
\text { reading and pedagogic needs of } \\
\text { the learning topic. }\end{array}$ \\
\hline
\end{tabular}

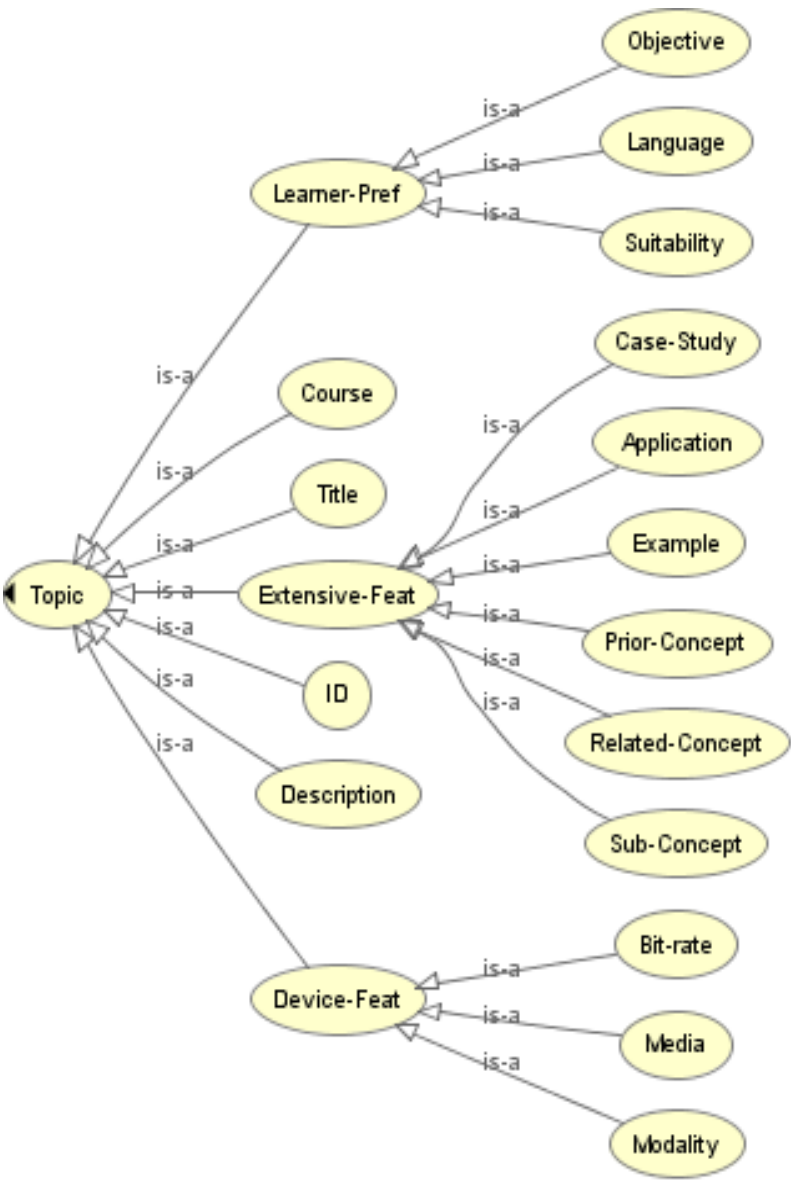

Figure 2. Layout of different core classes in CALRO ontology

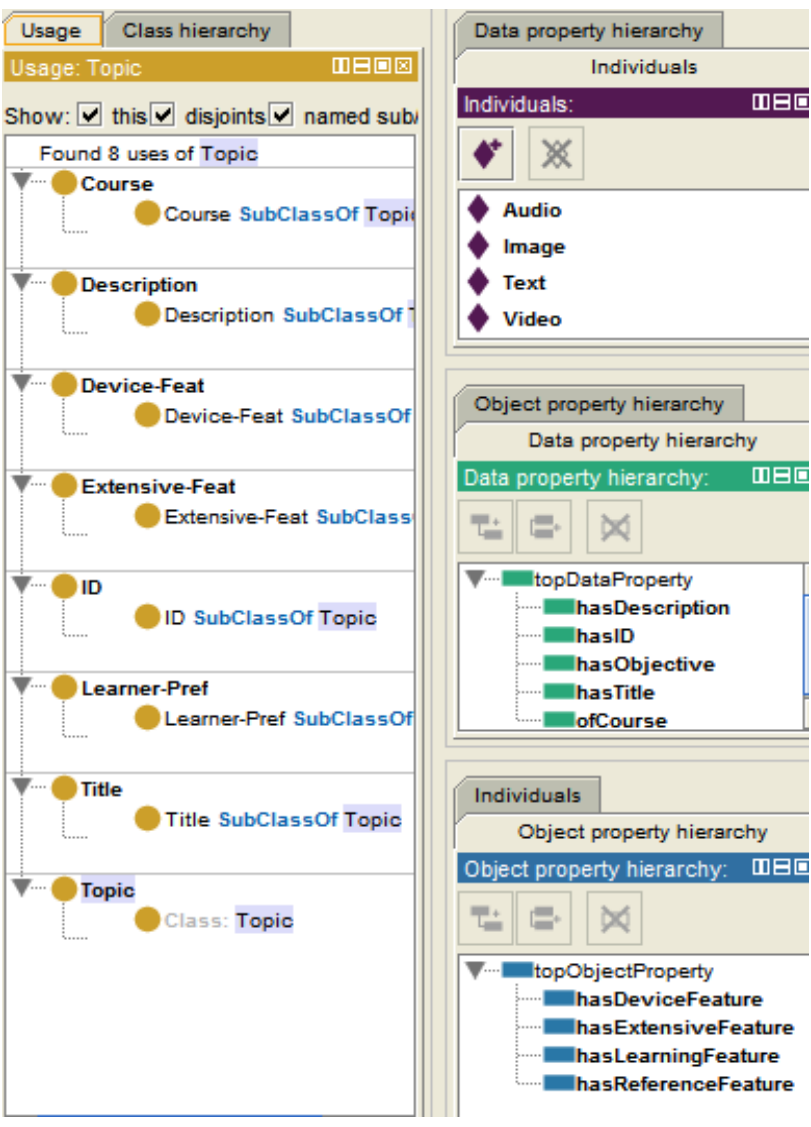

Figure 3. Properties and individuals of CALRO

\section{A. Formal representation of CALRO}

The Table II shows the partial OWL representation of the proposed CALRO ontology. The ontology consists of various classes and concerned relations along with the data type properties of topic.

TABLE II. OWL REPRESENTATION OF PARTIAL “CALRO"

\section{$<$ rdf:RDF}

xmlns:rdfs="http://www.w3.org/2000/01/rdf-schema\#"

xmlns:owl ="http://www.w3.org/2002/07/owl\#"

xmlns:rdf ="http://www.w3.org/1999/02/22-rdf-syntax-ns\#"

xmlns:xsd ="http://www.w3.org/2001/XLMSchema\#"

<owl:Ontology rdf:about="Resource Description using CALRO">

$<$ rdfs:comment $>$ Domain Ontology $</$ rdfs:comment $>$

$</$ owl:Ontology $>$

$<$ owl:ObjectProperty rdf:ID=" hasTitle">

$<$ rdfs:domain rdf:resource="\# Topic "/>

$<$ rdfs:range rdf:resource="\# Topic-Title "/>

$<$ rdfs:Datatype dc:title $=$ "graphs" $>$

$</$ owl:ObjectProperty $>$

$<$ owl:Class rdf:ID=" Media " $>$

$<$ rdfs:subClassOf rdf:resource="\# Device-Feat "/>

$</$ owl:Class $>$

$<$ owl:ObjectProperty rdf:ID=" hasMedia" $>$

$<$ rdfs:domain rdf:resource="\# Device-Feat " $>$

$<$ rdfs:range rdf:resource="\# Media "/>

$<$ rdfs:Datatype rdf:about=" \#XMLLiteral/">

$<$ owl:ObjectProperty $>$

$</$ rdf:RDF $>$ 


\section{B. Answering User Queries}

Here, we illustrate with an example how the CALRO is used to extract suitable topic based on learner context.

The system will locate the learning resource in the content repository, through considering the contextual information such as device capabilities and learner preferences. For example, consider the following scenario:

An English medium student wants to read about the topic "Graphs" in video format then the contextual information from the resource perspective is:

Learning topic $=$ Graphs;

Learning-Feat . Language $=$ English;

Device-Feat. Media $=$ Video;

The example query in Table III asks the SPARQL (SPARQL Protocol and RDF Query Language) processor for extracting the topic named "Graphs" which meets the given conditions.

TABLE III

THE “SPARQL” QUERY OF EXAMPLE SCENARIO

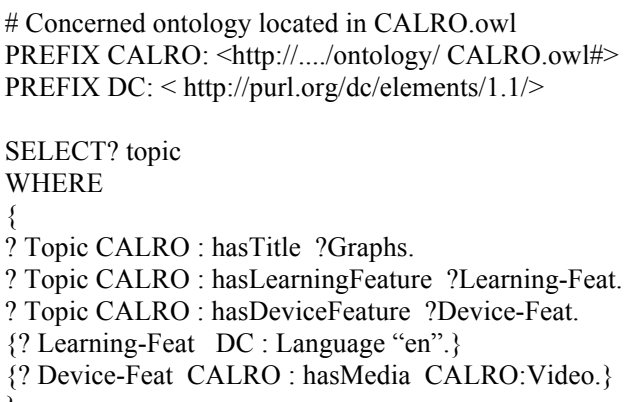

\section{PROposed AdAPTATION APPROACH}

The resource description ontology usage scenario is as shown in "Fig. 4", where the adaptation mechanism utilizes the CALRO to deliver précised and suitable content based on the learner context.

The current e-learning standards for educational metadata focus mainly on content centered approaches and models to search, exchange and reuse of learning resources [15]. The CALRO based description of learning contents is able to support the following features:

(1) Adaptive Presentation: as stated by Economides [11], the learning content can be presented in a variety of ways based on learner's prior preferences. The proposed ontology supports to deliver contents based on learner's learning style and preferences.

(2) Navigation support: Schmidt [16] mentioned that, the learning resources are not isolated, but formally or informally linked to one another. In the proposed ontological approach, the dependencies, topic similarities, sub topics, applications, etc. are linked together, so that it can help learner to navigate according to the conceptual relevance.

The CALRO ontology based resource description helps the adaptation system to identify the contents that correspond to the request and according to the device characteristics and user preferences.

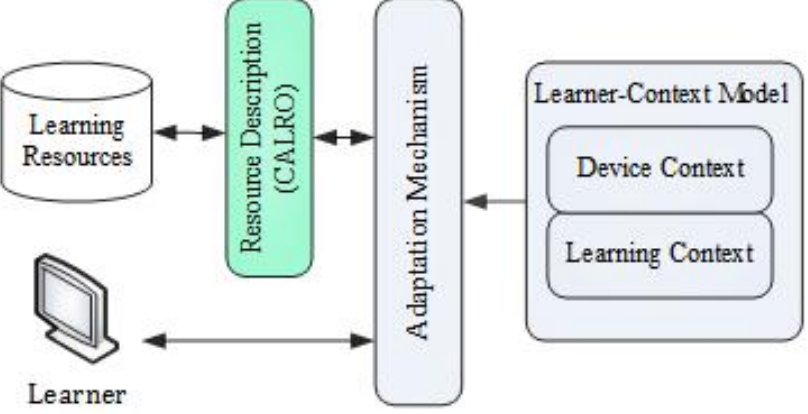

Figure 4. CALRO usage scenario

The objective of proposed course based e-learning application is to provide complete knowledge related to topics of the course, through delivering resources of different types such as definitions, exercises, case studies, etc. The well formed presentation and topic navigation environment makes the learner to access various resources related to learning concept.

"Fig. 5" shows the content presentation and navigation facilitates of the proposed approach. It relies on semantic based presentation of the course contents in order to improve the understandability of e-learner. Learner can refer to a wide range of different concepts that are concerned to learning topic. This approach facilitates the acquisition of new knowledge by the students through studying the related concepts even though they are not directly related to course content.

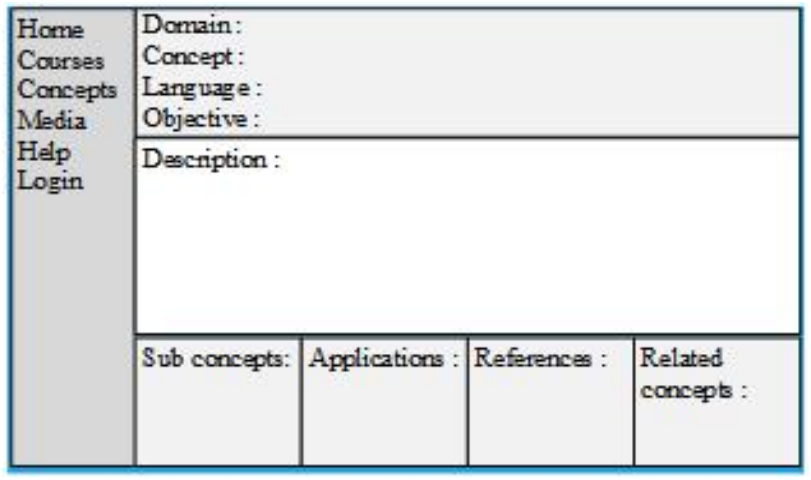

Figure 5. Proposed presentation and navigation screen structure

\section{A. Workflow behavior in context aware delivery}

The CALRO resource description ontology is useful mainly for context aware adaptive delivery of content in course based e-learning environment. The workflow behavior of the context aware adaptive delivery process consists of following steps and is depicted in "Fig. 6".

(1) First, the registration process has to be completed by the learner so that the learner is allotted with learner-id, which will be used to identify the learner.

(2) When learner's login process is completed, the system automatically detects the type of devicefeatures, through evaluating the "User Agent [17]" of HTTP request.

(3) Before starting to learn particular course contents, the learner needs to enter the preferences such as media, orientation of learning, etc. 
(4) The contextual information is stored under concerned learner's context-id. Based on contextual information and using CALRO ontological metadata (of resource information) the SPARQL returns the suitable learning content.

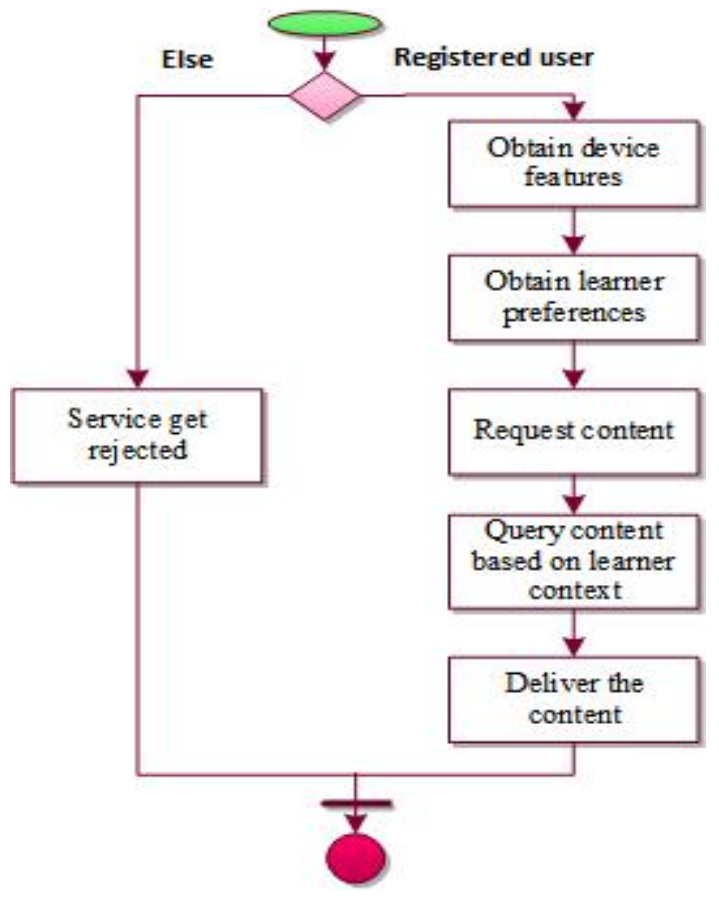

Figure 6. Workflow behavior

\section{CONCLUSION}

In context aware e-learning environments, using ontology for resource modeling allows us to deliver suitable and précised contents as per the domain specific context and preferences of e-learner. In this paper, we investigated various required features that are to be considered while developing resource description ontology. We believe that the proposed Context aware Adaptive Learning Resource Ontology (CALRO) enables the discovery of suitable learning resource based on device capabilities and learning style of e-learner. The extensive features enable the learner to extract and understand complete details of concerned learning topic.

As a future work, we aim to upgrade the existing distance e-learning system in our university to course based adaptive device independent system in order to enhance the readability and understandability of elearners, through adaptive interaction and presentation capabilities of proposed ontology. We believe that the current system should be enhanced in its adaptation capabilities through incorporating the proposed ontology.

\section{REFERENCES}

[1] Tretiakov, A. and Kinshuk, (2004) 'A unified approach to mobile adaptation of educational content', Proceedings of the IEEE International Conference on Advanced Learning Technologies (ICALT'04).

[2] Roy, N., Gu, T., \& Das, S. K. (2010). "Supporting pervasive computing applications with active context fusion and semantic context delivery". Pervasive and Mobile Computing, 6(1), 21-42. http://dx.doi.org/10.1016/j.pmcj.2009.10.002

[3] Devedzic, V. (2003) "Key issues in next-generation web based education", IEEE Transactions on Systems, Man, and Cybernetics, Part C, vol. 33, no. 3, pp. 339-349. http://dx.doi.org/10.1109/ $\underline{\text { TSMCC. } 2003.817361}$
[4] Laroussi, M. (2012). Ontology in Adaptive Learning Environment. In Workshop on Learning Technology for Education in Cloud (LTEC'12) (pp. 167-177). Springer Berlin Heidelberg. http://dx.doi.org/10.1007/978-3-642-30859-8 16

[5] Wilson, R. (2004). The role of ontologies in teaching and learning. JISC Joint Information Systems Committee Technology and Standards Watch Report TSW0402.

[6] Holohan, E., Melia, M., McMullen, D., \& Pahl, C. (2005). Adaptive e-learning content generation based on semantic web technology. Proceedings of Workshop on Applications of Semantic Web Technologies for e-Learning, Amsterdam, The Netherlands, pp. 29-36.

[7] Brusilovsky, P. and Vassileva, J. (2003) 'Course sequencing techniques for large-scale web-based education', Int. J. Continuing Engineering Education and Lifelong Learning, Vol. 13 , Nos. $1 / 2$, pp.75-94.

[8] Nejdl, W., Wolf, B., Qu, C., Decker, S., Sintek, M., Naeve, A., \& Risch, T. (2002, May). EDUTELLA: a P2P networking infrastructure based on RDF. In Proceedings of the 11th international conference on World Wide Web (pp. 604-615). ACM.

[9] Lassila, O., \& Swick, R. R. (1998). Resource Description Framework (RDF) model and syntax specification.

[10] Gasparini, I., Pimenta, M. S., Palazzo M de Oliveira, J., \& Bouzeghoub, A. (2010). Combining ontologies and scenarios for context-aware e-learning environments. In Proceedings of the 28th ACM International Conference on Design of Communication (pp. 229-236). ACM.

[11] Economides, A. A. (2009). Adaptive context-aware pervasive and ubiquitous learning. International Journal of Technology Enhanced Learning, Vol. 1, No 3, pp. 169-192, Inderscience. ISSN (Online): 1753-5263, ISSN (Print): 1753-5255.

[12] Almaoui, M., \& Plataniotis, K. N. (2005). Scalable e-learning multimedia adaptation architecture. In Image Analysis and Recognition (pp. 191-198). Springer Berlin Heidelberg. http://dx.doi.org/10.1007/11559573_24

[13] Siadaty, M., Torniai, C., Gašević, D., Jovanovic, J., Eap, T., \& Hatala, M. (2008, June). M-LOCO: An ontology-based framework for context-aware mobile learning. In Proceedings of the 6th International Workshop on Ontologies and Semantic Web for Intelligent Educational Systems collocated with the 9th International Conference on Intelligent Tutoring Systems, Montreal, Canada.

[14] Matthew Horridge, Simon Jupp, Georgina Moulton, Alan Rector, \& Robert Stevens. (2007, October 16). A Practical Guide To Building OWL Ontologies Using Protégé 4 and CO-ODE Tools. Edition 1.1. The University Of Manchester.

[15] Paramythis, A., \& Loidl-Reisinger, S. (2003). Adaptive learning environments and e-learning standards. In Second European Conference on e-Learning (pp. 369-379).

[16] Schmidt, A. (2005, October). Potentials and challenges of contextawareness for learning solutions. In Proceedings of the 13th Annual Workshop of the SIG Adaptivity and User Modeling in Interactive Systems, Saarbrücken, Germany. Retrieved on November (Vol. 16).

[17] UAProf(UserAgentProfile)Version20,2001:Availableat:http://open mobilealliance.org/wpcontent/uploads/2012/12/wap-248-uaprof20011020-a.pd

AUTHORS

Dr. V. Cyril Raj, Head, Dept. of Computer Science, Dr M.G.R University, Chennai, India; E-mail: hodcse@drmgrdu.ac.in

Dr. T. Ravi, Principal, Srinivasa Institute of Engineering \& Technology, Chennai, India; E-mail: travi675@yahoo.com

Kalla. Madhu Sudhana, Ph.D. Scholar, Dept. of Computer Science, St. Peter's University, Chennai, India; E-mail: kallamadhu1@yahoo.com

Submitted 02 August 2013. Published as re-submitted by the authors 11 February 2014. 